\title{
Urgences
}

\section{Textes d'enfants}

\section{Marie-Josée Paquette, Nathalie Jolicœur, Sylvain Côté, Stéphane Ruel, Yannick Sinclair et Bruno Morin}

Numéro 8, 4e trimestre 1983

Littérature jeunesse

URI : https://id.erudit.org/iderudit/025125ar

DOI : https://doi.org/10.7202/025125ar

Aller au sommaire du numéro

Éditeur(s)

Urgences

ISSN

0226-9554 (imprimé)

1927-3924 (numérique)

Découvrir la revue

Citer ce document

Paquette, M.-J., Jolicœur, N., Côté, S., Ruel, S., Sinclair, Y. \& Morin, B. (1983).

Textes d'enfants. Urgences, (8), 103-114. https://doi.org/10.7202/025125ar d'utilisation que vous pouvez consulter en ligne.

https://apropos.erudit.org/fr/usagers/politique-dutilisation/ 


\section{Textes d'enfants}



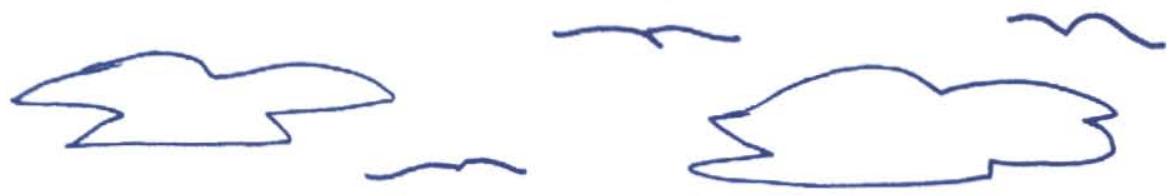

\section{LE PRINTEMPS}

Le mois d'avril est revenu.

Le printemps est déjà là

Avec son beau lilas

Qui me tend les bras.

Son soleil rayonnant

Me réchauffe tout le dedans.

Je me réjouis aux belles choses du printemps.

Alors, tout le monde l'accueille en souriant.

II faut en profiter jusqu'à l'été.

Marie-Josée PAQUETTE

11 ans,

Ecole Notre-Dame de Pitié

Québec.

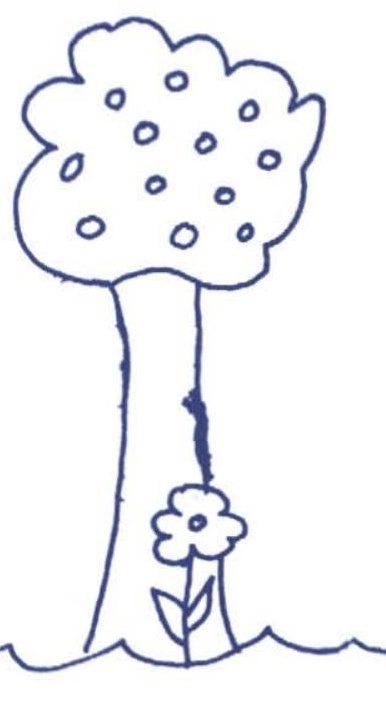




\section{MATHÉMATIQUES}

Toutes ces mathématiques...

Ce n'est pas toujours comique!

Sans cesse calculer,

Sans jamais s'arrêter.

Toujours bien lire ses questions Pour que ce soit toujours bon.

Toujours additionner, Sans jamais se tromper.

Nathalie JOLICOEUR, 12 ans,

Ecole Notre-Dame de Pitié Québec

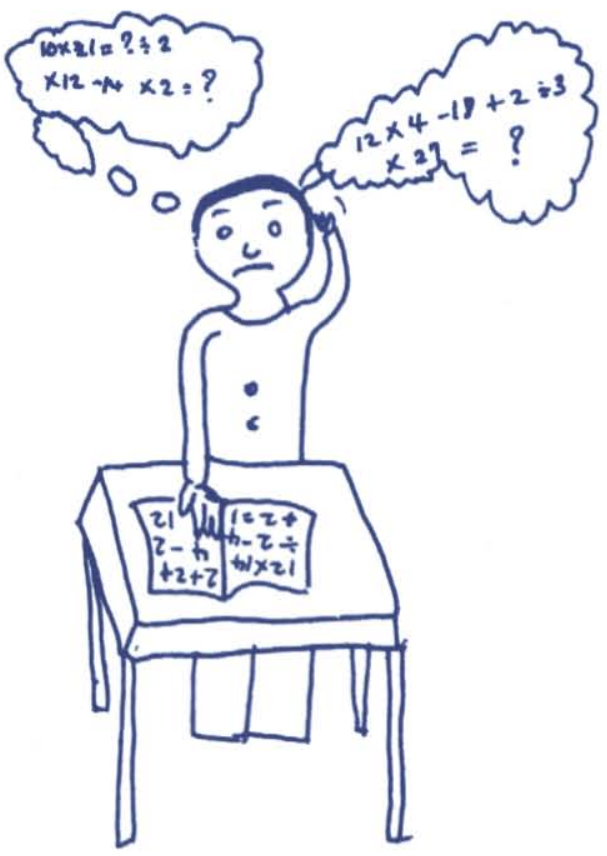


Dans la forêt étrange, c'est la nuit.

Dans les branchages bleus,

Des papillons volètent dans le soir

Puis s'en vont dans le noir.

Nathalie JOLICOEUR,

12 ans

Ecole Notre-Dame de Pitié

Québec
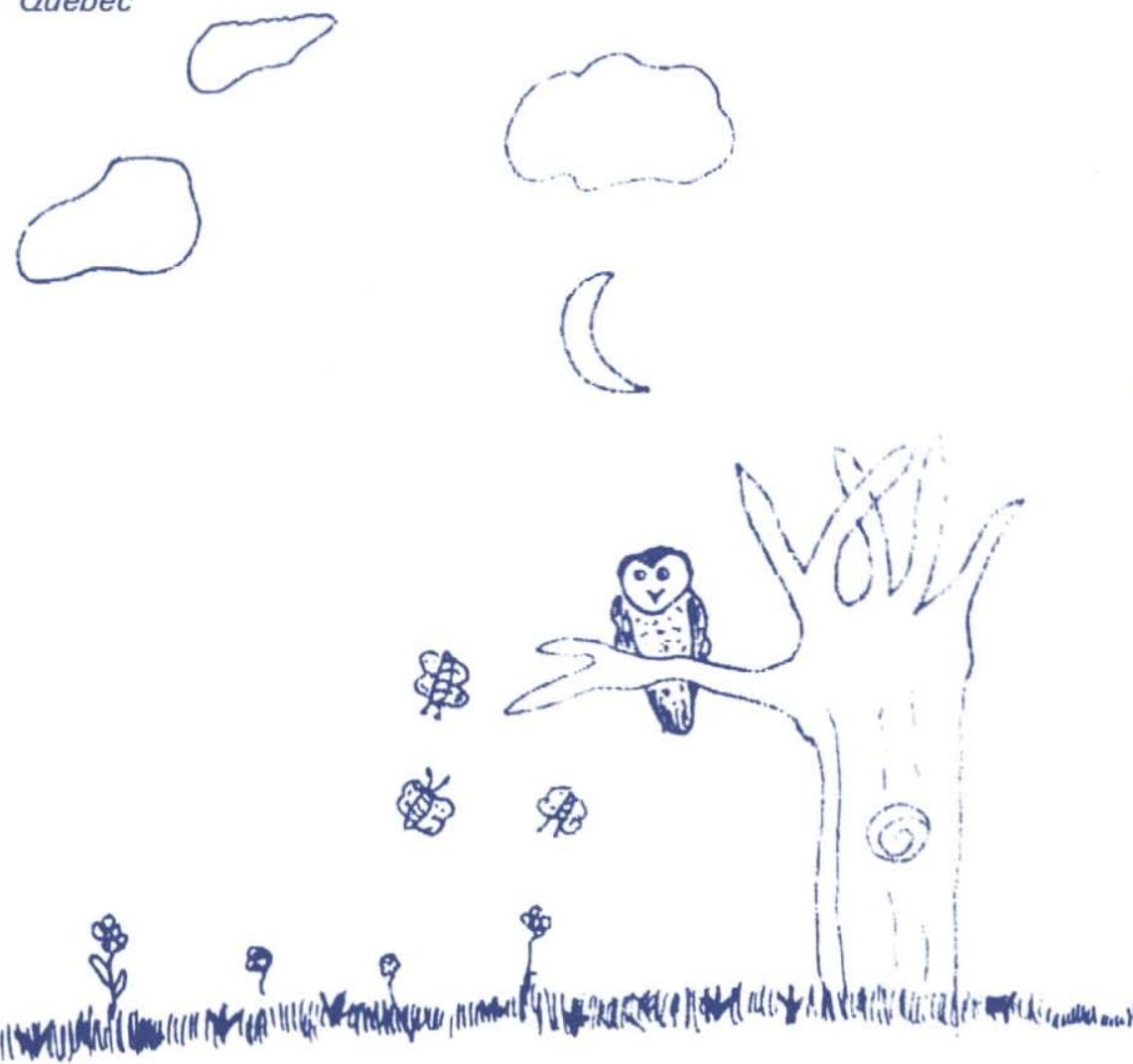

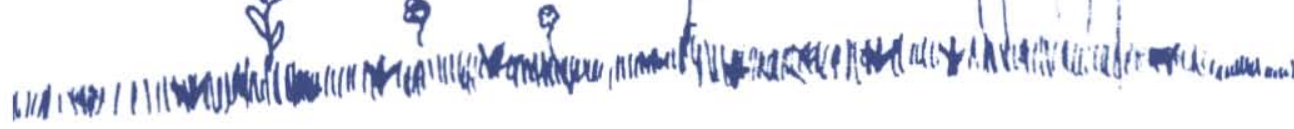




\section{Trois gros Indiens}

Un gros, deux gros, trois gros Indiens du Nord se promenaient avec leurs grosses bottes d'ours. Ils avaient chacun un fusil bien solide. Ils allaient chasser l'ours quand ils voulaient dans les sentiers. Parfois, ils voyageaient sur les rivières; parfois, ils montaient leurs chevaux tout tachés de roux et ils chantaient: "Woupi! Woupi! Woupi!" Les trois gros Indiens chantaient et chantaient au son des tambours et ils chantaient tellement bien que les gens leur criaient "Woupi!". Avant de se coucher, ils fumaient le calumet de paix. Bien au chaud dans leurs couvertures en peau, ils chantaient d'une voix grave, la chanson que leur avait appris leur père, un des plus grands chefs de la région "Woupi! Woupi!"

\section{Stéphane Ruel}

\section{C.S. Vallée de la Matapédia}
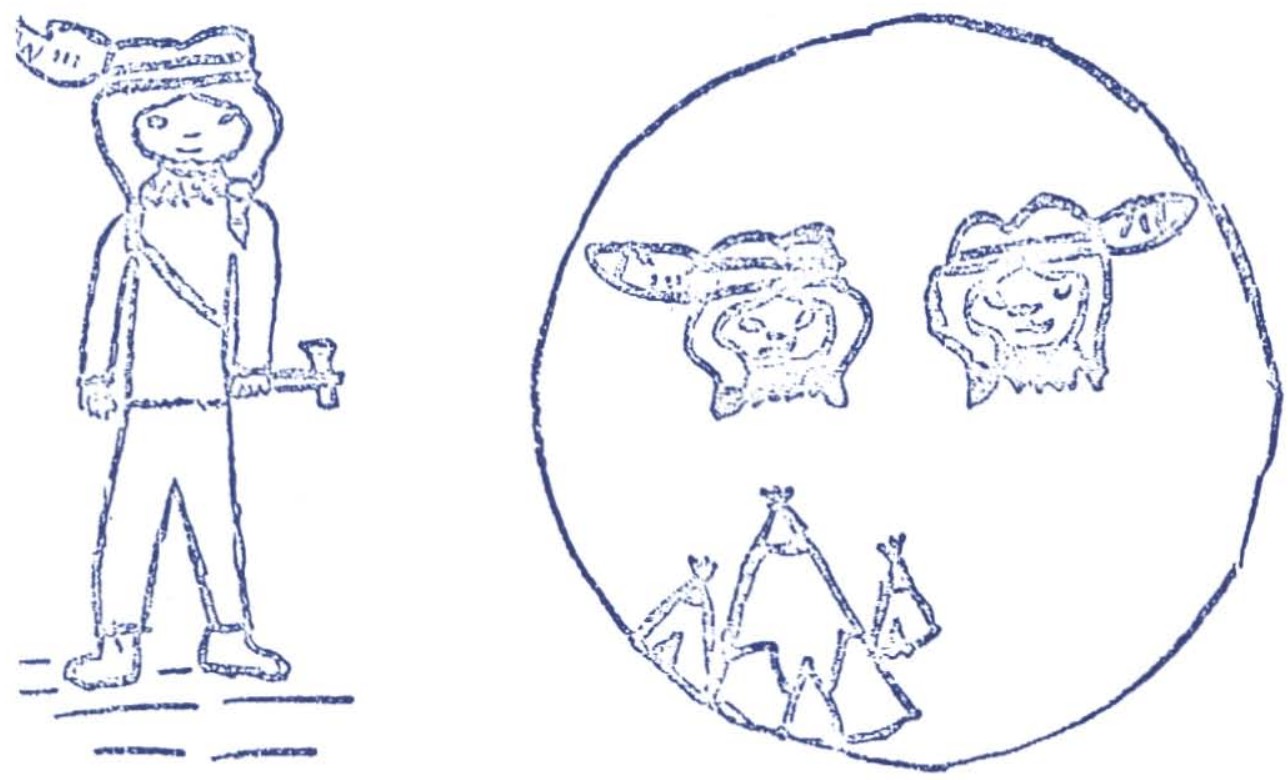


\section{Une drôle de mission}

Deux êtres bizarres appelés Plutot et Luneau habitent dans un laboratoire de la planète Jupiter.

Ces deux extra-terrestres ont pour but de s'emparer d'une nouvelle fusée conçue par les terriens.

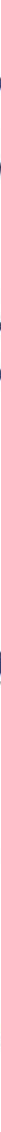


Un jour, Plutot dit à Luneau:

- J'ai une mission importante à te confier.

- C'est quoi ta mission? dit Luneau.

- Viens dans ma bulle spatiale, je vais t'expliquer.

- Voilà, le travail consiste à dérober la nouvelle fusée X-003, inventée par les visages pâles.

- Comment on vole ça une fusée?

- Mais voyons, Luneau, réfléchit un peu! Tu vas descendre sur la terre avec notre vieille fusée et pendant la nuit, tu n'auras qu'à les échanger. Par la suite, tu remontera sur la planète Jupiter.

- D'accord!
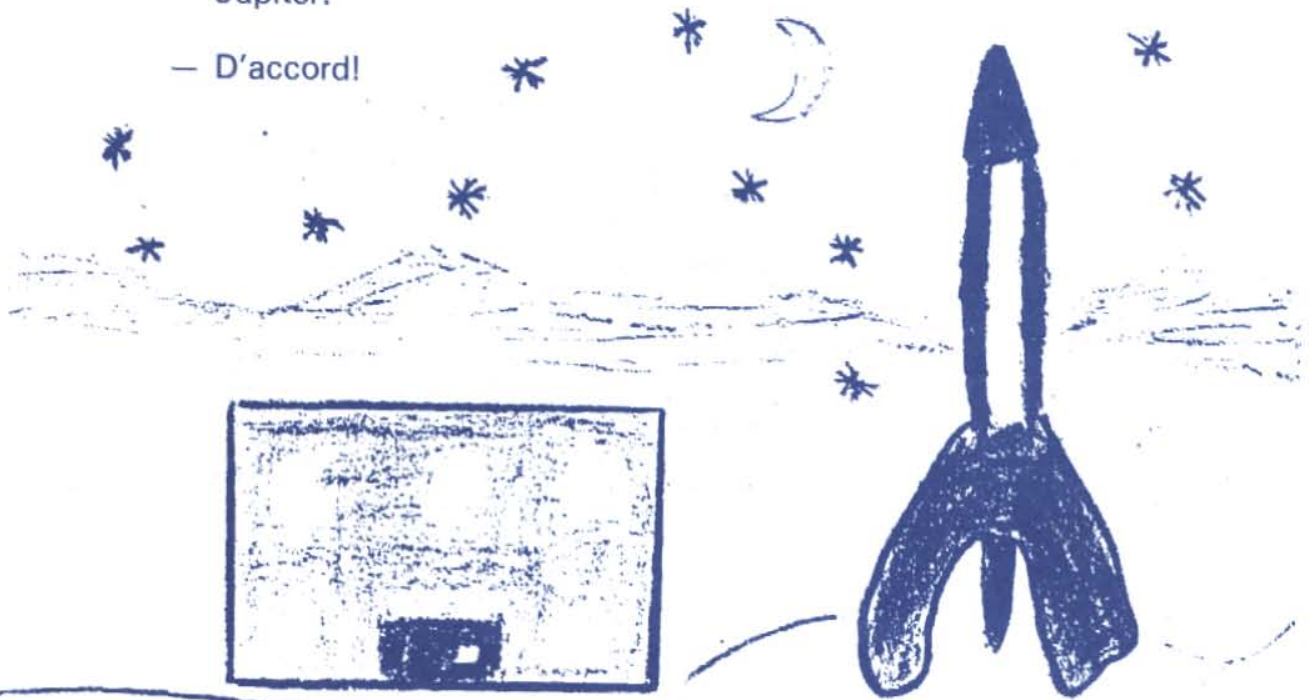
Le lendemain matin, Luneau saisit tout ce qui est comestible et décolle.

A mi-chemin, une petite conversation s'engage entre Luneau et le robot ZZZ. Ils parlent de la fête de Noël.

Arrivés sur la terre, ils sortent prudemment. Les deux créatures sont émerveillées. Un si grand nombre de lumières décorent la Nassa!

- Je suis curieux d'aller voir ce qui se passe, dit Luneau.

- En avant, reprend $Z Z Z Z$, nous y trouverons peut-être la fameuse fusée!

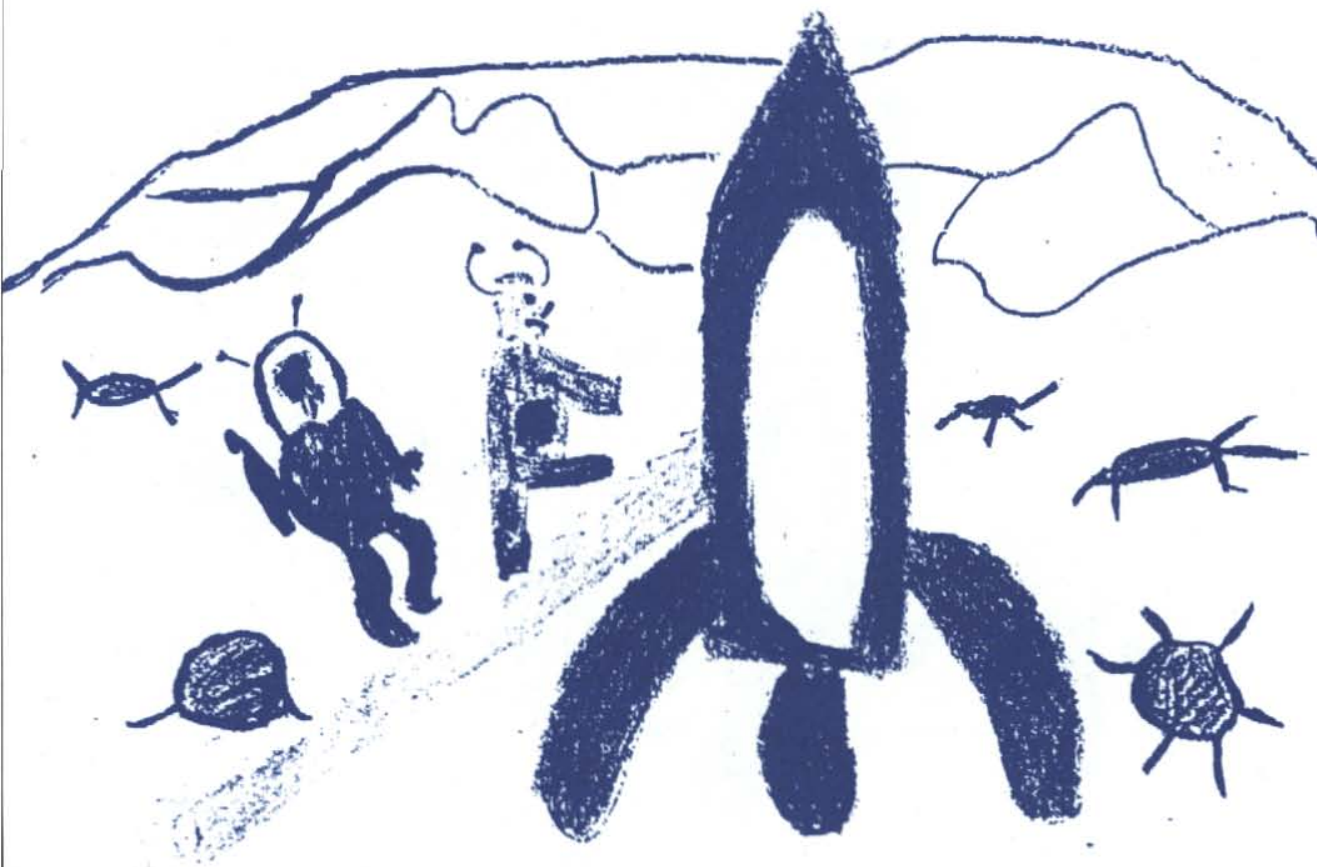


Les voici, s'avançant devant une grande porte.

- As-tu vu, dit ZZZ? On dirait qu'ils célèbrent la fête dont tu m'a tant parlé. Comment tu l'appelles déjà?

- Noël! Noël!

- Oui, je crois que c'est cela.

- ZZZ, regarde au bout du mur, on dirait la fusée.

- Tu as raisons. C'est elle. Comme elle est belle et lumineuse!

- Je cours à la fusée avertir Plutot.

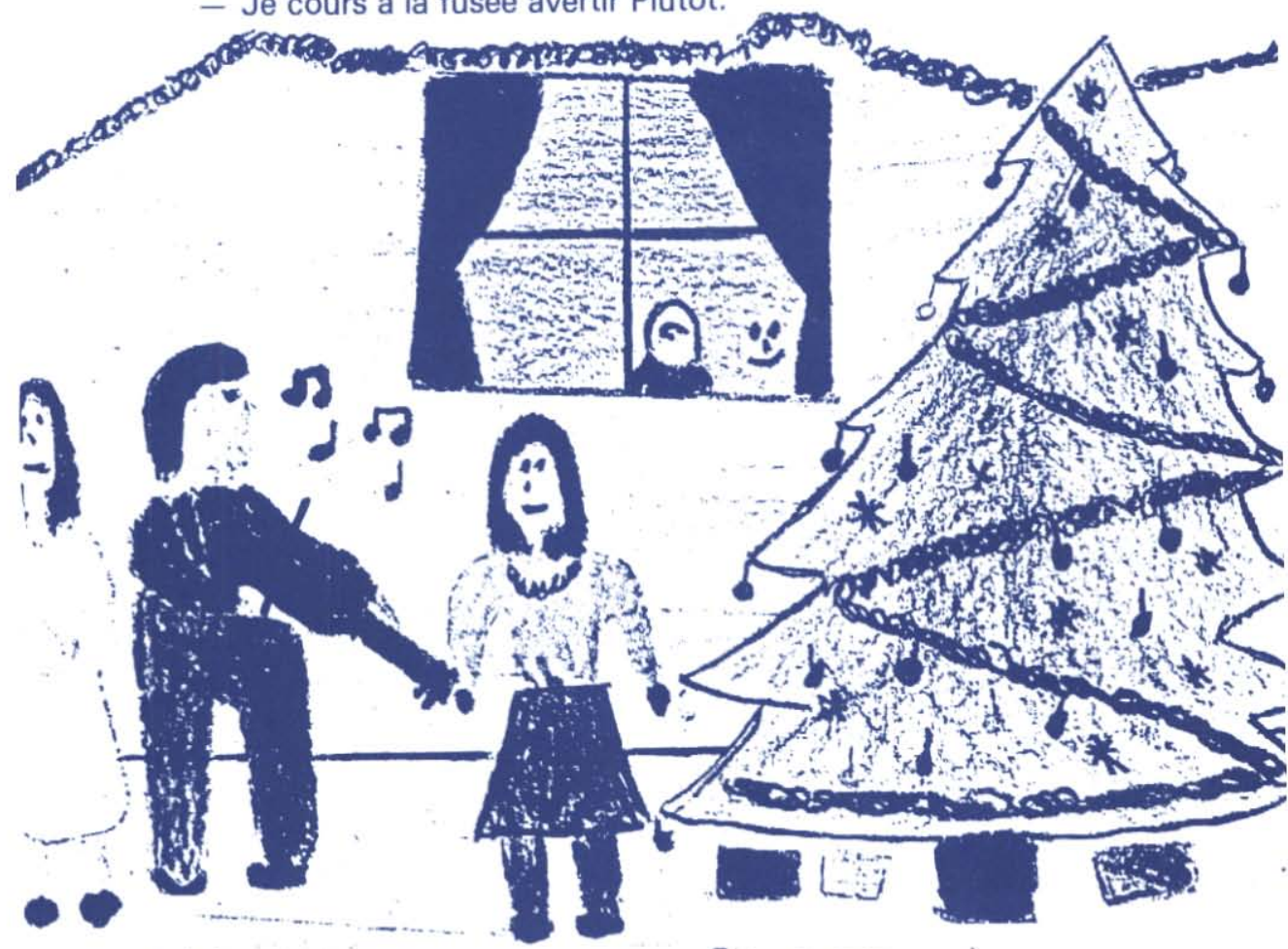




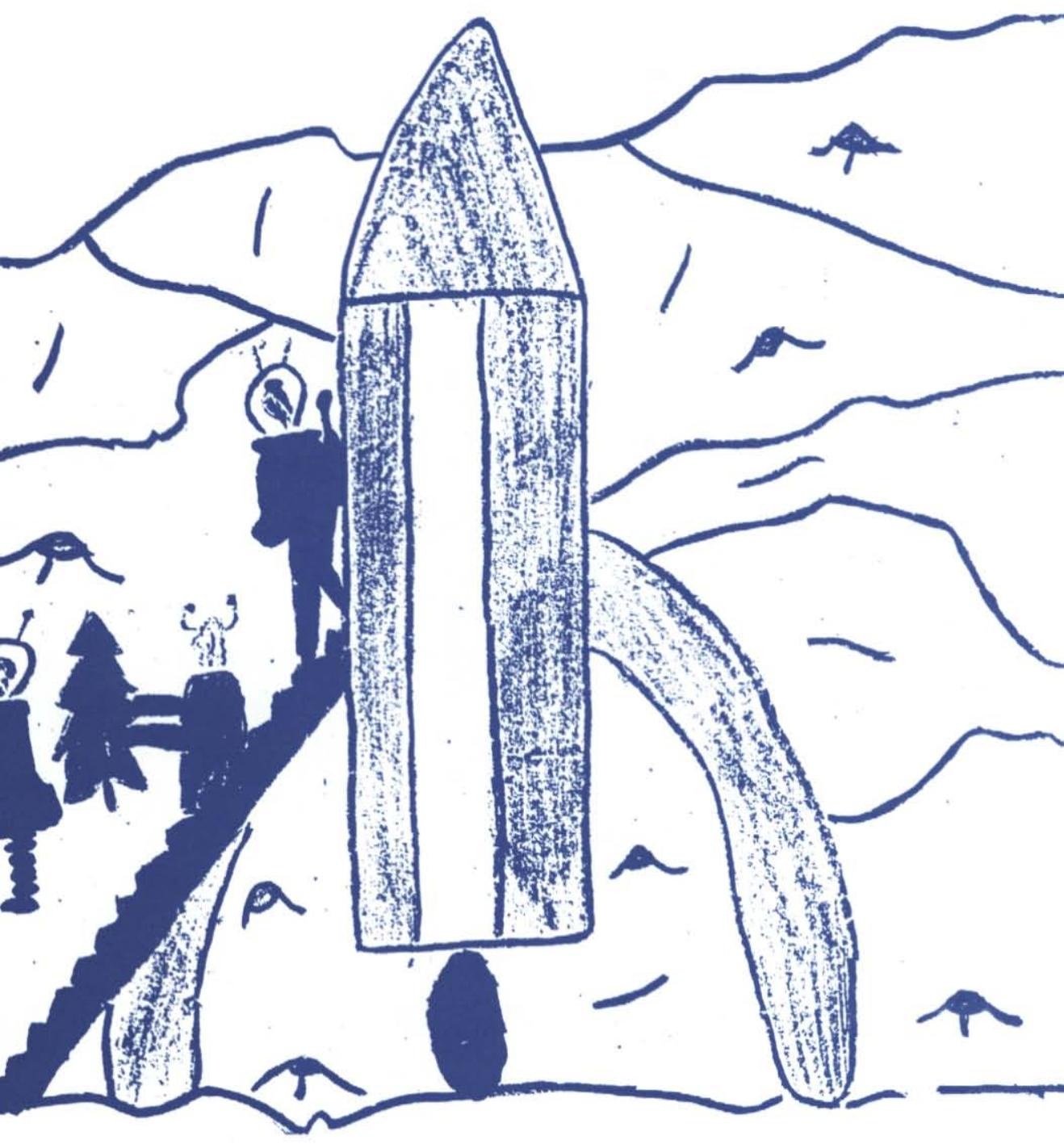


- Ici l'agent ZZZ, répondez agent 007.

- Agent 007 à l'écoute! Que voulez-vous?

- Nous avons réussi à repérer la nouvelle fusée.

- Bravo! vous êtes de courageux espions et bon retour.

Ils s'introduisent à l'intérieur de la Nassa. Ils s'emparent du sapin de Noël, se croyant en possession de la remarquable fusée, ils retournent vers leur vaisseau spatial et filent sur Jupiter.

A peine ont-ils touché Jupiter, que Plutot accourt I'air mécontent.

- Par Jupiter, qu'avez-vous fait de la nouvelle fusée?

- Patience! Patience! ZZZ apporte notre merveilleuse trouvaille!

- ZZZ revient tenant précieusement le résultat de leur mission.

- Ah! non! s'écrit Plutot! Est-ce possible? Vous n'êtes que des naiifs. J'en perds mes antennes! Ce n'est pas une fusée, c'est un arbre de Noël. Les terriens vous ont bien eus.

\section{Yanick Sinclair}

Bruno Morin

$6 e$ année

C.S Vallée de la Matapédia. 